\title{
Criminologie
}

\section{À propos des illégalismes privilégiés. Réflexions conceptuelles et mise en contexte}

\section{Fernando Acosta}

Volume 21, numéro 1, 1988

Entre les mailles de la loi : pollution, accident de travail, faute professionnelle

URI : https://id.erudit.org/iderudit/017256ar

DOI : https://doi.org/10.7202/017256ar

Aller au sommaire du numéro

\section{Éditeur(s)}

Les Presses de l'Université de Montréal

ISSN

0316-0041 (imprimé)

1492-1367 (numérique)

Découvrir la revue

Citer cet article

Acosta, F. (1988). À propos des illégalismes privilégiés. Réflexions conceptuelles et mise en contexte. Criminologie, 21(1), 7-34. https://doi.org/10.7202/017256ar

\section{Résumé de l'article}

The resolution of conflicts in a society can be seen as a complex network of interactions between various relatively autonomous official control systems. The place occupied by the criminal law as well as its role within this network are larg e -ly determined by the nature of its relations with the other control systems. Based on these theoretical premises, developed in recent socio-juridical studies, this article advances a number of conceptual proposals aimed at clarifying a question that has been the subject of theoretical and political controversy in the field of criminology for about half a century. It is the question of the exclusion, total or partial, of certain particular forms of illegalities from penal intervention. The principal characteristic of these illegalities lies in the fact that they have a broad range of forms of control (civil, administrative proceedings and particularly amicable arrangements). The control of illicit activities in five areas of social life examined in the second part of the study (the business world, public administration, public health, the environment and health and safety in the workplace) show this characteristic very clearly. In conclusion, the author advances a number of arguments that tend to illustrate the profoundly illusory nature of any attempt at control of the above-mentioned illicit activities by intervention of the criminal law.
Ce document est protégé par la loi sur le droit d'auteur. L’utilisation des services d’Érudit (y compris la reproduction) est assujettie à sa politique d'utilisation que vous pouvez consulter en ligne.

https://apropos.erudit.org/fr/usagers/politique-dutilisation/ 


\section{À PROPOS DES ILLÉGALISMES PRIVILÉGIÉS RÉFLEXIONS CONCEPTUELLES ET MISE EN CONTEXTE*}

Fernando Acosta**

The resolution of conflicts in a society can be seen as a complex network of interactions between various relatively autonomous official control systems. The place occupied by the criminal law as well as its role within this network are large ly determined by the nature of its relations with the other control systems. Based on these theoretical premises, developed in recent socio-juridical studies, this article advances a number of conceptual proposals aimed at clarifying a question that has been the subject of theoretical and political controversy in the field of criminology for about half a century. It is the question of the exclusion, total or partial, of certain particular forms of illegalities from penal intervention. The principal characteristic of these illegalities lies in the fact that they have a broad range of forms of control (civil, administrative proceedings and particularly amicable arrangements). The control of illicit activities in five areas of social life examined in the second part of the study (the business world, public administration, public health, the environment and health and safety in the workplace) show this characteristic very clearly. In conclusion, the author advances a number of arguments that tend to illustrate the profoundly illusory nature of any attempt at control of the above-mentioned illicit activities by intervention of the criminal law.

La problématique de la gestion différentielle des illégalismes ${ }^{1}$ par l'appareil pénal a été considérablement enrichie, au cours des toutes dernières années, par l'émergence d'un nouveau secteur de recherche qui s'interroge, d'une part, sur la place du pénal dans l'ensemble de systèmes de régulation formels (en d'autres termes, systèmes de droit

* Les propositions conceptuelles dont fait état cet article ont pris naissance dans le cadre d'une recherche, actuellement en cours, sur les atteintes à la vie en milieu de travail au Quebec, subventionnée par le Conseil de recherches en sciences humaines du Canada

** Professeur au Département de criminologie de l'Université d'Ottawa, Ottawa, Ontario, K1N 6N5.

1. C'est sans doute à Michel Foucault (Surveiller et punir, Paris, Gallimard, 1975) que l'on doit l'introduction, dans les textes criminologiques, du terme d'«illégalismes». Son utilisation, de plus en plus fréquente, dans cette discipline a fini par rendre parfois obscur le sens précis dans lequel on le prend. Dans le but d'éviter d'éventuels malentendus, il convient de préciser que l'emploi de ce terme dans le cadre de cet article désigne tout acte qui contrevient aux lois ou à l'ensemble de prescriptions qui en découlent. Les questions qui seront ici soulevées justifient, nous semble-t-il, amplement l'usage d'un terme d'aussi large portée.

Criminologie, $\mathrm{XX}, 1$ 
positif) et, de l'autre, sur les rapports qu'entretiennent entre eux ces différents systèmes de régulation ( $\mathrm{y}$ compris le droit pénal ${ }^{2}$ ) dans le cadre d'une société. Quelques-uns des travaux les plus récents de Pierre Lascoumes ${ }^{3}$ témoignent de l'importance et de l'urgence d'investir ce champ complexe de relations juridiques qui se nouent dans un cadre plus large (et sûrement encore plus complexe) de rapports économiques, sociaux et politiques. Autrement dit, ce que l'on cherche, entre autres, c'est à dépasser un certain penchant "pénalocentriste» ${ }^{4}$ qui a marqué quelques travaux qui ont ouvert la voie à cette problématique. J'entends par là le résultat, isolé de l'une ou l'autre, ou combiné, des deux tendances suivantes. Premièrement, de celle qui consiste à centrer à un tel point l'effort d'analyse sur l'activité de l'appareil pénal que l'on finit par perdre de vue $i$ ) les mécanismes qui ont permis, à un nombre incalculable de conflits, analogues à ceux qui déclenchent la répression pénale, d'éviter cette forme d'intervention et ainsi connaître une autre modalité de résolution (poursuites civiles, administratives, ententes à l'amiable, etc.) et $i i$ ) le fait que l'exclusion d'un certain nombre de conflits de la voie pénale, alors qu'il s'y trouvent déjà (ex. : abandon des poursuites par la Couronne), peut être due au détournement de ces conflits vers ces autres voies.

La tentative de combler les lacunes théoriques et empiriques laissées en cours de route par ces deux tendances, constitue, pour ce nouveau courant de recherche, le point de départ d'une problématique nouvelle où le pénal, malgré ses particularités et, surtout, les conséquences spécifiques qu'il engendre, est vu comme un système de régulation, un champ normatif parmi d'autres (comme, par exemple, les champs civil, administratif, commercial, etc.). Le problème de recherche qui se trouve ainsi posé est celui de comprendre comment ces différents champs normatifs, de même que les différentes pratiques d'intervention qui en découlent, se partagent et, ce faisant, gèrent l'univers des rapports sociaux conflictuels. En dépit des connaissances plutôt embryonnaires

2. Nous désignons, par ce terme, l'ensemble de prescriptions qui relèvent aussi bien du droit criminel que du droit pénal statutaire.

3. Voir, en particulier, i) P. Lascoumes, «Sanction des fautes ou gestion des illegalismes : l'hétérogénéisation du droit pénal. Un exemple de répression de la fraude fiscale», Revue interdisciplinaire d'études juridiques, I0, 1983, pp. 125-156; ii) P. Lascoumes, «Pénal répressif et/ou pénal restitutif. La place du pénal dans les stratégies de régulations administratives", in Aubusson de Cavarlay, Bruno et al., le Pénal en pre mière ligne ou en dernier ressort, Paris, CESDIP, 1984, pp. 221-401; iii) P. Lascoumes, Des erreurs, pas des fautes, Paris CESDIP, 1986.

4. Voir P. Lascoumes, op. cit., note 3, ii, pp. 233-238. Soulignons, cependant, que nous utilisons ce terme dans un sens qui ne coïncide pas exactement avec celui que luj attribue Lascoumes. 
dont on dispose en cette matière, une constatation importante semble s'imposer sans grande difficulté : soit que le droit pénal est loin de pouvoir revendiquer un contrôle hégémonique du champ de conflits que l'on pourrait logiquement lui attribuer sur la base des seuls énoncés qui constituent son armature discursive. Or, s'il est vrai que cette constatation peut trouver attestation empirique dans le domaine large des illégalismes populaires, il me semble qu'elle acquiert une importance et un intérêt grandissants lorsqu'on la situe dans le domaine de ce que j'appelle les illégalismes privilégiés.

C'est ce que je tenterai de montrer dans les pages qui suivent, en rappelant sommairement, dans un premier temps, deux différentes manières - proposées dans la littérature criminologique des dernières années - d'aborder la question de l'exclusion, partielle ou totale, de certaines formes d'illégalismes du champ de l'intervention pénale. Ces premières remarques nous permettront d'expliciter davantage le cadre théorique dans lequel nous situons nos propos. Je tiens, d'autre part, à préciser que malgré les réserves que l'on puisse avoir, et que j'ai moimême, à l'égard de l'emploi du terme impunité pénale pour désigner ce que, dans une formule condensée, on pourrait appeler exclusion pénale, j'opterai pour son utilisation dans ce texte afin d'éviter les ambiguïtés de sens que cette demière formule pourrait sans doute créer.

En deuxième lieu, je proposerai les éléments essentiels de définition de ce que j'entends par illégalismes privilégiés et j'essaierai, par un exercice de mise en contexte, de confronter ces éléments à un certain nombre de connaissances, fort inégales, dont on dispose à l'heure actuelle en cette matière. Par ces propositions, qui forment le noyau de cet article, je cherche à réunir sous un même toit conceptuel des cadres empiriques qui se sont constitués soit à l'intérieur, soit en marge, soit entièrement à l'extérieur du champ d'investigation de la criminologie.

En troisième lieu, j'examinerai brièvement les principales dimensions que l'on a privilégiées dans la littérature criminologique, pour aborder la question de l'impunité pénale des illégalismes que nous étudions. En guise de conclusion, je soulèverai un certain nombre d'arguments qui cherchent à indiquer les problèmes sérieux que pose tout projet de criminalisation des conduites qui relèvent des contextes examinés dans la seconde partie de ce texte.

\section{DEUX THÈSES SUR L'IMPUNITÉ PÉNALE}

Sans avoir mérité une attention soutenue de la part des auteurs en criminologie, la question de l'impunité en matière pénale fut abordée 
de manières diverses dans la littérature scientifique de cette discipline. En se limitant aux contributions datant des vingt dernières années, il est possible d'identifier deux grandes thèses par lesquelles l'on a essayé de saisir les éléments essentiels de cet objet.

La première, très en vogue au cours des années $60-70$, envisage l'impunité pénale comme un attribut de classe. En d'autres termes, le fait que les conflits impliquant des membres des fractions dominantes ne connaissent que rarement des suites pénales, témoignerait avant tout d'un «biais de classe» dont seraient «victimes», en partie, les législateurs, mais, surtout, les opérateurs des agences chargées de l'application de la loi pénale. On y voit sans grandes difficultés le reflet, au plan de la conception du droit pénal et de son application, de la théorie instrumentale de l'État qui a largement dominé les propositions des courants radicaux nord-américains ${ }^{5}$. Thèse de combat par excellence, et tant que l'on reste à ce seul niveau il y a des mérites que l'on oublie souvent de lui reconnaître, on doit cependant admettre qu'elle cache mal son penchant fonctionnaliste dans la mesure où elle réduit la problématique de l'impunité pénale à une simple question de distorsions (apparemment rectifiables) au plan de l'application de la norme pénale. L'accent résolument volontariste de cette thèse reste encore visible même dans les rares incursions faites par ses partisans dans le domaine de la criminalisation primaire. La production de la norme pénale y est souvent présentée comme un processus à sens unique à travers lequel la classe bourgeoise s'assurerait de façon massive et à tout jamais sa domination sur la classe laborieuse. Notons, par ailleurs, que le fait que l'armature conceptuelle employée par les tenants de cette thèse soit empruntée à la théorie marxiste a conduit plus d'un critique à y voir l'archétype mal achevé d'une théorie marxiste du crime et de son contrôle. Ce n'est pas le propos de cet article de faire la démonstration du caractère hautement abusif d'une telle assimilation. Nous tenons cependant à souligner que cette thèse a connu effectivement une certaine fortune, dans le cadre des débats criminologiques des années 70 , et que dans ce sens elle a sûrement contribué à maintenir la problématisation théorique de l'impunité pénale à l'intérieur de limites assez étroites.

La deuxième thèse, dont l'embryon se trouve sans doute dans les résultats des recherches pionnières, de la fin des années 30 , sur la cri-

5. Le lecteur trouvera plusieurs exemples de cette tendance dans les écrits parus, tout particulièrement au cours des années 70, dans la revue Crime and Social Justice, porte-parole du groupe de criminologues radicaux de Berkeley. 
minalité des affaires ${ }^{6}$, propose une représentation typologique des modes de règlement des conflits en les distribuant sur un continuum de pratiques et de mesures diverses qui vont de l'entente à l'amiable à l'emprisonnement ferme, en passant par les sanctions administratives et les réparations civiles, entre autres. L'impunité pénale prend, dans un tel schéma, la forme de tout recours à un mode de règlement de conflit autre que ceux de nature pénale, alors que ceux-ci étaient, du moins en principe, juridiquement plausibles. Les premiers travaux de Lascoumes sur la délinquance d'affaires en France proposent, tour à tour, différentes versions plus sophistiquées de ce modèle de base? ${ }^{7}$. Le problème majeur que pose cette forme de représentation de l'impunité pénale - et Lascoumes lui-même est le premier à s'en rendre compte - tient essentiellement à la raison suivante: c'est qu'en étalant les divers modes de règlement des conflits sur un continuum de pratiques et de mesures, on suggère, d'une part, l'idée d'une gradation logique entre ces divers modes, de l'autre, l'idée d'une rationalité qui présiderait au choix, dans chaque cas concret, du mode le plus adéquat et efficace d'intervention. Or, le peu que nous savons à l'heure actuelle sur la gestion extrêmement complexe des contentieux pénalement atypiques (comme c'est le cas, par exemple, des litiges dans le domaine des affaires) suffit pour nous convaincre qu'il n'y a pas une logique gestionnaire d'ensemble en cette matière mais, plutôt, des rapports tantôt de collaboration, tantôt d'exclusion, entre différentes logiques institutionnelles de prise en charge.

Les reformulations successives de cette thèse, dans l'ensemble de travaux de recherche de Lascoumes, ont abouti à ce que l'on pourrait considérer comme étant, à la fois, une variante de la thèse originale et (surtout) le noyau d'une problématique nouvelle. Cette problématique élargit considérablement les possibilités d'approche de l'objet impunité pénale dans la mesure où elle l'inscrit dans une large réflexion sur l'organisation et le fonctionnement de la gestion différentielle des illégalismes dans une société. C'est dans le cadre de cette problématique que nous essayons d'orienter nos recherches actuelles en cette matière et, bien entendu, les propositions conceptuelles qui suivent. Avant d'y

6. Nous pensons ici aux travaux pionniers de Sutherland et pas nécessairement à ceux de ses disciples, souvent orientés dans la ligne de ce qu'ils appellent la «criminalité occupationnelle». Voir, entre autres, E. Sutherland, White-Collar Crime, New York, Holt, Rinehart \& Winston, 1949.

7. La meilleure version de ce modèle se trouve probablement dans P. Lascoumes, J.C. Weinberger, «Delinquenza di affaristi e problemi d'affari», la Questione criminale, 4, (1), 1978, pp. 63-97. 
arriver, il convient cependant de présenter, dans ses traits essentiels, la manière par laquelle nous la concevons.

La gestion des illégalismes dans une société peut être représentée sous la forme d'un réseau d'interactions entre divers systèmes normatifs relativement autonomes. Cette forme de représentation nous permet d'identifier, dans l'ensemble de rapports conflictuels gérés par les divers systèmes de droit, deux zones fort distinctes. En se servant de la figure 1, ci-après, qui illustre, dans ses traits essentiels, une situation d'interaction relativement banale, nous pouvons identifier la première zone (parties ombragées de la figure) comme étant représentative de ce

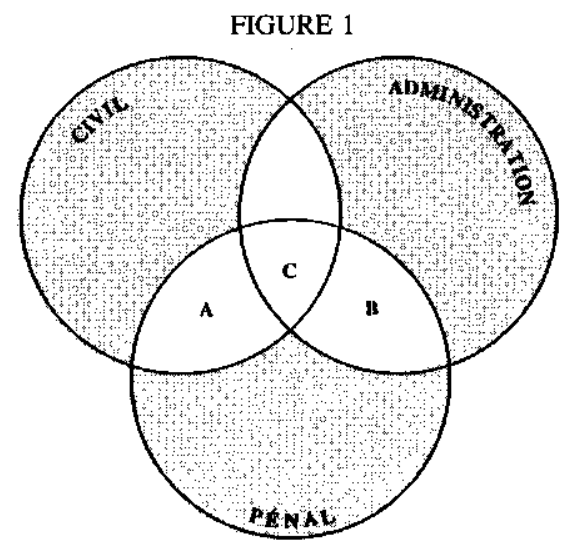

que nous pourrions appeler les illégalismes typiques d'un système normatif, c'est-à-dire ceux qui, du moins en principe, relèvent en exclusivité d'un système normatif donné. À défaut d'entente entre les parties en conflit, l'annulation d'un contrat de vente, pour vice rédhibitoire, ne peut prendre la forme d'une action judiciaire que dans le cadre des normes qui relèvent du champ du droit civil. De mêmé, prenons un deuxième exemple, la fabrication de fausse monnaie ne peut faire l'objet d'une prise en charge judiciaire qu'à la lumière des préceptes relatifs au droit pénal. Dans un cas comme dans l'autre, nous dirions que l'événement à l'origine de chacune de ces situations conflictuelles n'est (potentiellement) juridiquement significatif que dans les limites du code de lecture de la réalité que propose un système normatif en particulier, et seulement ce système. Au plan de la norme substantive, il constitue alors ce que j'appelle un illégatismes typique du système en question.

La deuxième zone (parties non ombragées de la figure 1), est constituées par trois sous-zones distinctes: pénal/civil (A) pénal/admi- 
nistratif (B) et pénal/civil/administratif (C). C'est, bien entendu, cette zone qui retient particulièrement mon attention car, contrairement à celle que l'on a examinée précédemment, il est ici question d'un nombre important de situations de conflit dont la signification juridique particulière n'est pas au premier abord évidente. En d'autres termes, leur éventuelle qualification, au plan juridique, peut, du moins en principe, se faire à la lumière des énoncés de plus d'un système normatif, ils sont plurisignificatifs, ces conflits, aux yeux du droit. C'est, entre autres, en vue de saisir le caractère équivoque (au sens fort du terme) de ces réalités conflictuelles et, plus précisément, de leur qualification juridique et éventuelle prise en charge (judiciaire ou autre) que je propose le terme d'illégalisme privilégié. Bien qu'ils soient fortement (mais non exclusivement) associés à des pratiques auxquelles n'ont accès que les membres des fractions dominantes de la société, on s'en doutait bien, il serait cependant hâtif de conclure que c'est en raison d'une telle fatalité que j'applique l'épithète de privilégié à ces illégalismes. Ce qui ne veut pas dire que je ne vois pas une certaine utilité dans cette coïncidence.

\section{LES ILLÉGALISMES PRIVILÉGIÉS : REMARQUES CONCEPTUELLES}

Par illégalismes privilégiés, je désigne l'ensemble de qualifications juridiques applicables à des situations conflictuelles, par ailleurs fort diverses, qui possèdent les trois caractéristiques essentielles suivantes.

i) Au plan juridique: l'appréhension de ces conflits peut se faire à la lumière de différents champs normatifs, notamment le droit pénal, le droit civil et, tout particulièrement, le droit administratif ${ }^{8}$. Il est donc de la nature de ces illégalismes, comme nous l'avons, en d'autres termes, déjà suggéré, de pouvoir s'insérer dans plus d'un registre juridique à la fois, d'emprunter plus d'un type de qualification à la lumière du droit positif.

ii) Au plan événementiel: les événements constitutifs de ces situations conflictuelles gardent une homologie frappante avec des événements dont la qualification juridique et l'éventuelle prise en charge sont de compétence exclusive du droit pénal. Il est ici question aussi bien de dommages pécuniaires d'importance plus que considérable que des atteintes (directes ou indirectes) à la vie dont les conséquences sont, dans bien des cas, extrêmement graves.

8. Au sujet de la place, de plus en plus importante, occupé par le droit administratif dans les règlements des conflits voir op. cit., note 3 , ii. 
iii) Au plan des règlements des conflits: ces situations conflictuelles disposent, en principe, d'un large éventail de modes de règlement (ententes à l'amiable, avertissements, sommations, sanctions disciplinaires, amendes statutaires à caractère symbolique, réparations civiles, etc.). L'utilisation effective d'un mode de règlement plutôt qu'un autre dépend cependant de plusieurs facteurs qui varient, d'ailleurs, sensiblement d'un genre de situation à l'autre (opportunité de l'intervention, nature des rapports entre le contrevenant et ceux qui sont chargés de l'application d'une loi ou d'un règlement particulier, connaissances et représentations, de la part d'éventuelles victimes, de la nature des conflits où elles se trouvent impliquées, ressources matérielles et humaines des organismes de contrôle, état des rapports, collaboration mutuelle? concurrence? conflit ouvert? entre des organismes de contrôle relevant de différents paliers de l'administration publique, et j'en passe). C'est essentiellement en raison de cette large disponibilité de modes de résolution et, également, de l'extrême complexité qui semble caractériser la dynamique de leur mise en ceuvre, que je qualifie de privilégiés les illégalismes dont il est ici question. Nous sommes donc très loin, me semble-t-il, des horizons bouchés qui confinent, la plupart du temps, la résolution si souvent simpliste et expéditive des illégalismes populaires9.

À ces remarques conceptuelles, il convient d'ajouter un certain nombre d'illustrations qui cherchent, dans la mesure du possible, à mettre à contribution les connaissances (d'ailleurs fort inégales) dont on dispose à l'heure actuelle en cette matière. C'est ce que propose le tableau 1, ci-après, que nous avons construit à partir des trois caractéristiques énoncées plus haut. En l'examinant, le lecteur devra être bien averti que, s'il est vrai que cet exercice d'illustration fut pensé en tenant compte, le plus possible, des contextes canadien et québécois, il n'en demeure pas moins que bien des lacunes empiriques ont dû être remplies à l'aide de données provenant de recherches menées dans d'autres pays. Ce handicap ne nous semble cependant pas porter un prejudice sérieux à l'usage premier auquel nous destinons ce tableau, dans le cadre de cet article, qui est celui d'être un moyen de représentation/visualisation, à travers des formes concrètes, des trois dimensions qui nous apparaissent essentielles à la conceptualisation de ce que

9. Nous ne prétendons aucunement, par cette affirmation, que la compréhension de l'organisation et du fonctionnement de la répression pénale constitue une tâche banale, ce qui serait du reste une formidable hérésie. Nous affirmons seulement que les mécanismes de résolution des conflits imposés par la loi en matière pénale (et, en particulier, criminelle) s'avèrent, lorsque comparés à ceux dont disposent d'autres formes de conflits, d'un manque d'imagination parfois ahurissant. 
nous entendons par illégalismes privilégiés. II n'est cependant pas exclu, au contraire, que l'ensemble de propositions qu'il avance puissent également être considérées comme autant d'hypothèses de travail des recherches qu'il reste à faire.

Dans sa première section horizontale, champ événementiel, nous avons disposé cinq «domaines» de la vie sociale (et, dans certains cas, disons plutôt domaines de la vie quotidienne) pouvant générer les conflits à l'origine de ces illégalismes. Il s'agit, on s'en doute bien, d'une énumération qui ne prétend aucunement être exhaustive ${ }^{10}$. Il est question, en ce qui concerne les deux premiers domaines (monde des affaires, au sens strict d'activités financières et économiques, et administration publique), de litiges de nature strictement patrimoniale. On aura sans doute ici reconnu les points d'ancrage de deux secteurs bien établis de recherche dans le champ des sciences sociales : les investigations en matière de délinquance d'affaires ${ }^{11}$ et de corruption politicoadministrative $^{12}$ en témoignent d'ailleurs amplement. Par contre, les litiges en cause dans les trois autres domaines portent sur des atteintes à la vie, c'est-à-dire des dommages causés aussi bien à l'intégrité physique des individus qu'à un certain nombre de conditions nécessaires à leur existence. On y trouve les domaines suivants: $i$ ) santé publique (pour l'essentiel, soins médicaux et production et mise en marché de médicaments), ii) environnement (l'activité visée ici étant, bien enten$\mathrm{du}$, le rejet de substances polluantes dans le milieu ambiant), et, finalement, iii) santé et sécurité au travail (i.e. l'importance accordée à la salubrité et à la sécurité physique dans les conditions de travail offertes par les patrons à leurs employés).

Dans la deuxième section du tableau, champ des systèmes normatifs, nous trouvons les catégories de droit positif pouvant être mises à contribution en vue d'une qualification juridique des conflits issus de chaque domaine en particulier. Compte tenu de l'usage auquel se destine ce tableau nous n'incluons dans cette section que des catégories classiques de droit positif, ce qui implique, par conséquent, l'omission de toute une série d'autres textes mineurs, à caractère normatif (codes de déontologie, lignes directrices gouvernementales en matière de

10. Il est au moins deux autres domaines, celui du maintien de l'ordre et celui de la circulation automobile, qui en principe, semblent pouvoir être inclus dans le cadre de ces considérations. Certaines de leurs particularités, dont l'examen dépasse largement le propos de cet article, nous empêchent cependant de le faire pour l'instant.

11. Pour un excellent compte rendu des études entreprises dans ce champ, voir P. Lascoumes, op. cit., note 3, iii.

12. Voir F. Acosta, «La corruption politico-administrative : émergence, constitution et éclatement d'un champ d'études», Déviance et société, 9, (4), 1985, pp. 333-354. 
TABLEAU I

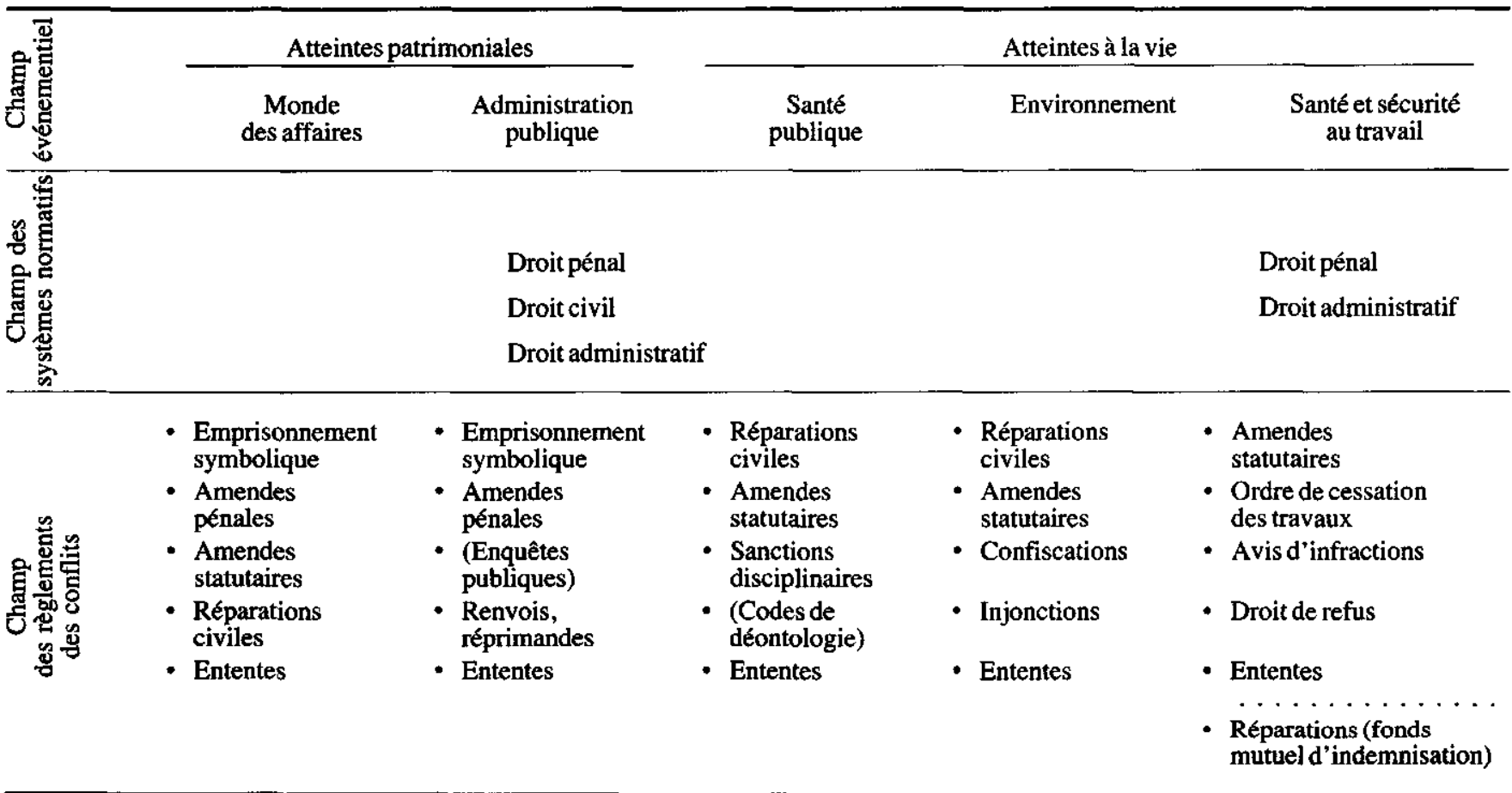


conflit d'intérêt, etc.), dont la réelle fonction régulatrice, dans certaines circonstances précises, n'est pas à dédaigner, comme du reste l'on verra dans la prochaine section du tableau.

Dans sa troisième et dernière section, champ des règlements des conflits, le tableau 1 présente, de façon sommaire, les modalités dominantes de résolution des litiges propres à chacun des domaines considérés. Ces modalités, comme on peut facilement le constater, débordent souvent le cadre normatif à partir duquel ces conflits peuvent être juridiquement qualifiés. Ceci s'explique $i$ ) dans les cas des sanctions disciplinaires, des congédiements et des demandes d'exonération, par la mise en garde que nous venons de faire au paragraphe précédent; $i i$ ) dans les cas des ententes à l'amiable, par le fait que les règles et contingences auxquelles elles se trouvent soumises ne sont pas, à peu d'exceptions près, prévues dans des textes légaux. Soulignons, également, que c'est dans le seul but de simplifier les tâches, en matière de présentation graphique, que nous disposons ces différentes modalités de règlement en échelles. Il ne faudrait pas y voir la suggestion d'une quelconque gradation réelle de sévérité de mesures et, moins encore, d'un quelconque parallélisme avec une échelle de gravité des illégalismes propres à chaque domaine. Ces «échelles» peuvent servir à indiquer, tout au plus, ce que l'on pourrait provisoirement appeler les «modalités extrêmes», en termes de complexité des procédures, des règlements de conflits qui prévalent dans chaque domaine. (Un exemple: la résolution d'un litige, en face à face, par la voie d'une entente où chaque partie réussit à faire admettre à l'autre, du moins partiellement, le bien-fondé de ses intérêts, apparaît à tous moins ritualiste et contraignante que la prise en charge, du même litige, par une cour criminelle. Un contre-exemple : une enquête peut s'avérer presque aussi contraignante et, à coup sûr, plus ritualiste qu'une procédure plus ou moins expéditive devant une instance criminelle). Entre ces extrêmes, de nombreuses permutations sont possibles.

Dressons maintenant un certain nombre de commentaires sur chacun des domaines figurant au tableau 1.

\subsection{MONDE DES AFFAIRES}

On ne compte plus, depuis les recherches pionnières de Sutherland dans le domaine du «white collar crime»13, les démonstrations empiriques de la thèse de l'exclusion systématique des illégalismes d'affaires de la voie du règlement pénal. À peu de différences près, les

13. Voir, entre autres, E. Sutherland, op. cit., note 6. 
nombreuses recherches (américaines, pour la plupart, et françaises) qui ont étudié le fonctionnement des filières de contrôle en ce domaine convergent remarquablement vers une même constatation: soit que ces illégalismes sont définis et traités, dans l'immense majorité des cas, dans le cadre de procédures d'entente ou alors au niveau de contentieux à finalité essentiellement restitutive. Nul n'est cependant autorisé à tirer de ce fait la conclusion vicieuse que tous les illégalismes ainsi gérés auraient pu faire l'objet d'une prise en charge pénale. L'importance des résultats produits par ces recherches tient essentiellement au fait qu'ils mettent en relief une réalité qui est commune aux formes les plus diverses d'illégalismes privilégiés, c'est-à-dire qu'elles disposent d'un éventail de modalités de règlements dont l'un des effets serait vraisemblablement celui de faire obstacle à la mise en cuvre des modalités les plus contraignantes à l'intérieur de l'ensemble d'interventions possibles, la répression pénale, tout particulièrement. Néanmoins, ceci n'implique aucunement que la mise à l'écart de la solution pénale, y compris la criminalisation, constitue une règle absolue. Le recours à ce mode de résolution de conflits demeure en effet possible dans le domaine des illégalismes d'affaires, comme en témoignent d'ailleurs un certain nombre d'études, y compris des recherches québécoises, qui se sont penchées sur cette question ${ }^{14}$. Mais s'il est vrai que l'on peut faire appel aux amendes prévues pour des infractions statutaires ou des violations au Code criminel ou même à des peines (symboliques ou de courte durée) d'emprisonnement en vue de sanctionner la conduite des hommes d'affaires (affairistes, à vrai dire, pour la plupart), il n'en demeure pas moins qu'il est permis en même temps d'affirmer que $i$ ) la part de poursuites pénales impliquant des hommes d'affaires, à l'entrée du contentieux pénal, est insignifiante; cette part se réduit encore davantage au fur et à mesure que l'on avance dans le processus judiciaire $\left.{ }^{15} ; i i\right)$ de nombreuses modalités de mise en échec de la prise en charge pénale peuvent intervenir au cours des poursuites et, dans certains cas même, suite à une condamnation à une peine d'emprisonnement (il faudrait sans doute parler, dans ces cas, d' 'radoucissements

14. Voir, P. Lascoumes, G. Moreau-Capdeville, Justice pénale et délinquance d'affaires, Paris, Service d'études pénales et criminologiques, 1983; J.M. Rico et al., la Criminalité d'affaires au Québec, Université de Montréal, École de criminologie, 1981; C. Parent, le Contrôle social de la criminalité d'affaires, mémoire de mâ̂trise inédit, Université de Montréal, École de criminologie, 1982; F. Acosta, "De l'événement à l'infraction: le processus de mise en forme pénale», Déviance et société, $11,(1), 1987$, pp. $1-40$.

15. Voir P. Lascoumes, op. cit., note $14, i$. 
pénaux») $)^{16}$ et, finalement, iii) les moyens dont dispose un homme d'affaires pour neutraliser les effets de son passage par l'appareil pénal (le casier judiciaire étant sans doute le plus négatif d'entre eux) sont considérablement plus efficaces que ceux qui s'offrent à l'infracteur ordinaire, comme du reste l'ont bien démontré Landreville, Pires et Blankevoort ${ }^{17}$. Contrairement à ce que l'on pourrait du premier coup penser, il n'est pas tout à fait insensé de parler d'impunité pénale même lorsque l'intervention pénale a lieu.

\subsection{ADMINISTRATION PUBLIQUE}

La plupart des remarques faites ci-haut au sujet des illégalismes propres au monde des affaires semblent se transposer sans grandes difficultés au domaine de l'administration publique, et tout particulièrement aux sphères de l'administration publique occupées par des hauts fonctionnaires, des membres du personnel politique et des détenteurs de mandats populaires. Le fait que, à toutes fins pratiques, il n'y a pas de corruption politique (pour penser au cas de figure classique en cette matière) sans délinquance d'affaires, opération douteuse d'affaires ou, tout simplement, homme d'affaires à l'arrière-plan de la scène, mérite sans doute d'être pris en considération lors d'une éventuelle réflexion sur une telle symétrie. En donnant libre cours à l'imagination, il serait sans doute raisonnable de prétendre que la ressemblance de plus en plus étroite entre le mode de gestion des affaires, dans le secteur privé, et celui de la chose publique, dans les différents secteurs de l'appareil gouvernemental, aurait probablement entrainé, à la longue, la mise sur pied de pratiques de règlements de conflits, dans chacun de ces secteurs, qui finiraient tout compte fait par se ressembler. Toute tentative de trouver des parallélismes en cette matière devant, en principe, faire preuve de prudence, il convient de souligner au moins deux dissemblances importantes entre ces deux domaines, que la réalité juridique canadienne et certaines recherches québécoises illustrent bien, en ce qui

16. Quelques-unes des poursuites criminelles qui ont été intentées contre des administrateurs publics et des hommes d'affaires, dans le sillage de l'affaire de corruption politique a Ville d'Anjou, au Québec, constituent des exemples parfaits de ce que nous entendons par «adoucissements pénaux». Un de ces administrateurs, pour n'en doruer qu'un exemple, qui fut condamné à 18 mois d'incarcération, n'est resté en fait que trois semaines en prison. Libéré pour des raisons de santé, il a attendu pendant quelques mois sa libération conditionnelle définitive dans une maison de convalescence dirigée par sa femme. Voir F. Acosta, op. cit., note 14, $i v$.

17. Voir P. Landreville et al., les Coats sociaux du système pénal, rapport de recherche, Université de Montréal, École de criminologie, 1982; A. Pires, Stigmate pénal et trajectoire sociale, thèse de doctorat inédite, Univérsité de Montréal, École de criminologie, 1983. 
à trait à l'organisation et au fonctionnement de leurs mécanismes respectifs de contrôle.

La première de ces dissemblances se trouve au plan de la loi pénale, qui définit les illégalismes de la sphère de l'administration publique de manière sensiblement moins équivoque qu'elle ne le fait pour ceux qui ont cours dans le milieu des affaires. Rappelons, en effet, que le Code criminel, dans sa partie III, consacre six de ses articles à l'institution d'infractions dont le modus operandi suppose nécessairement la participation d'un fonctionnaire public (art. 108, 109, 110 et 112) ou, alors, qui ne peuvent être commises que par des fonctionnaires publics (art. 111 et 117). Les écarts du monde des affaires, lorsqu'on les porte devant les cours criminelles, ne prennent forme juridique recevable, dans bien des cas, qu'à l'aide de véritables prodiges herméneutiques autour de l'énoncé légal de la fraude, à l'article 338 du même Code.

Deuxième dissemblance, qui vient d'ailleurs relativiser ce que nous venons de dire, le recours effectif à la loi pénale, dans le domaine des illégalismes des hauts membres de l'administration publique, semble peu probable, du moins au Québec, grâce en bonne partie au rôle joué par les commissions d'enquête en cette matière. C'est ce qui explique d'ailleurs le fait que nous ayons décidé, en encourant le risque de faire sursauter plus d'un lecteur, d'inclure cette procédure parmi les modalités de règlements des conflits propres à ce domaine. Un certain nombre de recherches portant sur le fonctionnement des enquêtes publiques, menées au Québec au cours des dernières années ${ }^{18}$, montrent en effet que les résultats qu'elles produisent peuvent soit $i$ ) prêter à des fins éminemment politiques et idéologiques, sans grande répercussion (ou, souvent même, aucune) au plan juridico-pénal, soit ii) être utilisés comme des conditions de possibilité de poursuites pénales dont la portée réelle est plus que relative. Or, l'on constate facilement que, dans un cas comme dans l'autre, le seul fait de déclencher et de garder active (ce qui n'est pas toujours chose facile) une enquête publique constitue déjà, pour ceux et celles dont les agissements furent, à travers cette démarche, étalés au grand jour $i$ ) une punition suffisamment sévère pour faire paraître juste la décision (gouvernementale) de ne pas les traîner en cour criminelle, ou $i i$ ) si jamais des poursuites sont intentées, une punition dont l'importance sera prise en considération, lors des calculs des sentences, en vue de réduire le plus possible les peines à leur imposer.

18. Voir, entre autres, J.P. Brodeur, la Délinquance de l'ordre. Recherches sur les commissions d' enquête I, Montréal, Hurtubise HMH, 1984; F. Acosta, op. cit, note 14, iv. 


\subsection{SANTÉ PUBLIQUE}

Tout indique que le domaine du contrôle des atteintes à la vie par des actes posés par des professionnels du champ médical ou, alors, par des pratiques intégrantes du processus de production et la mise en marché de produits pharmaceutiques, constitue un terrain on ne peut plus propice aux hypothèses et aux évaluations impressionnistes. C'est donc avec beaucoup de prudence que l'on doit considérer ce que propose notre tableau 1 dans la case réservée aux principales modalités de règlements de conflits dans le champ de la santé publique.

Malgré cet état de choses, que la pénurie flagrante d'études criminologiques en cette matière a sûrement aidé à édifier, il semble possible d'affirmer que le recours aux poursuites criminelles ou à l'emprisonnement en raison de violation à des normes statutaires, suite à des dommages causés par une intervention médicale ou par l'ingestion d'un produit pharmaceutique, est à toutes fins pratiques inexistant.

Quoique nous ne disposions pas de résultats de recherches canadiennes à ce sujet, il serait probablement instructif de rappeler ce que dit Braithwaite à propos des poursuites criminelles qui furent intentées contre les dirigeants de la compagnie allemande responsable de la découverte et de la mise en marché de la thalidomide. Cette drogue, nous rappelle Braithwaite, a causé des malformations chez environ 8000 enfants, dans 46 pays autour du monde et est à l'origine d'un nombre peut-être deux fois plus grand de morts à la naissance. Selon le criminologue australien, ces poursuites constituent «une exception à la tendance marquée de recourir au règlement civil plutôt que d'intenter des poursuites criminelles» qui prévaut en matière de litiges concernant des dommages occasionnés par des drogues dont les effets néfastes furent sciemment occultés par leurs fabricants ${ }^{19}$. Auteur de l'ouvrage sans doute le mieux documenté sur les pratiques illégales qui ont cours dans l'industrie pharmaceutique (son enquête porte sur les États-Unis, la Grande-Bretagne, l'Australie et, également, sur plusieurs pays du Tiers-Monde), Braithwaite soutient également qu'aucun dirigeant d'une importante compagnie pharmaceutique, aux États-Unis, n'est jamais allé en prison en raison d'une violation du Food, Drug and Cosmetic $A c t$, une loi qui jouit pourtant d'une solide réputation de sévérité, un peu partout au monde. Par contre, les poursuites civiles (dont le total des indemnisations ainsi que l'ensemble des frais sont, dans certains cas, entièrement assumés par des compagnies d'assurances), les

19. Voir J. Braithwaite, Corporate Crime in the Pharmaceutical Industry, London, Routledge \& Kegan Paul, 1984. 
amendes (dont les montants, parfois très substantiels, ne correspondent en fait qu'à un pourcentage insignifiant des sommes en jeu), et peutêtre surtout, les ententes (entre les agences de contrôle et les industries) semblent constituer les principaux piliers de la gestion «rationnelle et efficace» des conflits qui prime dans ce domaine.

En ce qui concerne les fautes du domaine de la pratique médicale, dont les modalités de prise en charge semblent se polariser autour des réparations civiles et des sanctions disciplinaires, nous convions notre lecteur à prendre connaissance de l'entretien avec le $D^{r}$ Bernard Lambert, expert médical, publié dans cette livraison ${ }^{20}$. Précisons, cependant, qu'il est possible qu'une analyse sectorielle de l'ensemble des diverses catégories professionnelles qui dispensent des soins médicaux puisse mettre en lumière d'autres modalités de règlements de conflits en ce domaine, comme, par exemple, dans le cas des fautes commises par des infirmières et infirmiers.

\subsection{ENVIRONNEMENT ET SANTÉ ET SÉCURITÉ AU TRAVAIL}

La question des règlements des conflits dans les domaines de l'environnement et de la santé et de la sécurité au travail a été traitée par Grandbois et Lippel, respectivement, dans le cadre de ce numéro de Criminologie ${ }^{21}$. C'est pourquoi je me limiterai ici à faire un certain nombre de remarques sur les propositions contenues dans le tableau 1, au sujet de chacun de ces domaines, en soulignant au passage quelques particularités que j'estime importantes.

Le champ de règlements des contentieux portant sur des questions environnementales présente une configuration assez semblable à celle que nous avons examinée à l'item précédent. À peu de différences près, on y trouve les mêmes modalités dominantes et, en particulier, les mêmes modalités dominantes extrêmes (ententes et réparations civiles) qui dominent l'horizon des règlements des conflits en matière de santé publique. Cette constatation immédiate appelle cependant quelques précisions.

Notons, en premier lieu, qu'il y a de bonnes raisons de croire que les poursuites civiles prennent, dans l'ensemble du contentieux environnemental, une place moins importante qu'elles n'occupent dans le

20. Voir, dans ce numéro de Criminologie «Entretien - L'expertise médicale en matière de faute professionnelle».

21. Voir, dans ce numéro de Criminologie, M. Grandbois, «Le droit pénal de l'environnement : une garantie d'impunité?»; K. Lippel, «Les victimes sans crimes : le traitement pénal des accidents de travail». 
domaine de la santé publique. La raison principale, nous semble-t-il, tient à la difficulté, en particulier pour le simple individu, d'identifier l'activité polluante qui est à l'origine d'un problème auquel il se trouve confronté. Ou encore pire: la difficulté pure et simple de savoir que c'est à une source de pollution qu'il pourrait, en principe, attribuer le problème qui l'afflige. Soulignons bien que ce n'est pas à l'épineuse question de l'établissement d'un lien de cause à effet entre les deux termes de la situation type ci-haut que nous nous référons, mais plutôt à une dimension strictement cognitive à défaut de laquelle il n'y a même pas conscience de litige. Cette constatation énonce, par ailleurs, ce qui est l'un des traits le plus insidieux des atteintes à l'environnement: le fait qu'elles peuvent souvent se constituer hors du champ de l'expérience quotidienne de ceux qui en subissent les effets, échappant ainsi à toute représentation sous forme conflictuelle. (J'admets volontiers que cette caractéristique n'est pas exclusive aux atteintes à l'environnement: on la trouve également dans le domaine de la santé publique, ce qui n'est pas surprenant, compte tenu des symétries que l'on peut retracer entre les pratiques nuisibles propres à chacun de ces domaines. J'estime cependant que ce trait marque d'une façon considérablement plus élargie l'ensemble de formes possibles de pollution de l'environnement.)

Deuxième précision. Au Canada, en général, tout indique que les ententes constituent le mode auquel on fait le plus souvent appel pour régler les litiges en matière d'environnement et, aussi, pour définir légalement et les litiges et la façon la meilleure de les résoudre ${ }^{22}$. Dans ce dernier cas, on parlera alors de négociation, c'est-à-dire de cette procédure qui constitue, selon Thompson (cité par Schrecker ${ }^{23}$ ), «l'essence même du processus de réglementation au Canada». C'est d'ailleurs ce même auteur qui nous rappelle que, en ce qui concerne l'environnement:

les principes de réglementation ne sont jamais énoncés de manière claire et précise, si ce n'est dans un sens purement symbolique. Les normes font l'objet de négociations incessantes entre les autorités et les entreprises visées, jusqu'au moment où ces dernières décident si elles vont s'y conformer... Les mécanismes coercitifs sont utilisés dans le seul cas d'un désaccord ultime, et encore, il peut s'agir alors d'une simple étape dans le cours de négociations interminables ${ }^{24}$.

22. À ce sujet, voir tout particulièrement T.F. Schrecker, l'Élaboration des politiques en matière d'environnement, document d'étude, Commission de réforme du droit du Canada, Ottawa, 1984.

23. T.F. Schrecker, op. cit., note 22.

24. T.F. Schrecker, op. cit., p. 9, note 22. 
On trouvera, entre autres, dans l'article de Grandbois sur le droit pénal de l'environnement ${ }^{25}$ d'excellents exemples de «produits» du processus de négociation dont nous parle Thompson.

Les propositions du tableau 1, dans le domaine de la santé et de la sécurité au travail, restent en principe circonscrites à la réalité québécoise. Néanmoins, si l'on se fie aux affirmations contenues dans un document récent de la Commission de réforme du droit du Canada sur la pollution en milieu de travail ${ }^{26}$, il y a des chances considérables que les propositions du tableau soient applicables, mutatis mutandis, à l'ensemble du Canada. C'est le cas, par exemple, de la règle générale selon laquelle il n'est pas possible d'intenter des poursuites en vertu du droit civil pour régler un conflit dans ce domaine. Dans le cas du Québec, comme le souligne bien Lippel27, il n'y a plus moyen de recourir à cette forme de résolution de litiges depuis l'avènement, en 1909, de la loi qui a introduit dans les mœurs de la province la théorie du risque professionnel ${ }^{28}$. C'est ce qui explique pourquoi on trouve seulement les catégories du droit pénal (criminel et statutaire) et du droit administratif dans le champ des systèmes normatifs (tableau 1) qui gouvernent les conflits en matière de santé et de sécurité au travail, communément appelés «accidents de travail».

Cette différence essentielle, entre ce domaine et les quatre autres qui figurent au tableau 1, explique à son tour la configuration particulière que prend la section réservée aux modalités dominantes de règlements de litiges en cette matière: on y trouve, en fait, aux positions extrêmes, les amendes pour des infractions statutaires et les réparations auxquelles ont droit tous les travailleurs couverts par la législation concernant les accidents de travail. Soulignons, cependant, que ces formes de règlement de conflits procèdent de deux logiques fort distinctes d'intervention. Dans le premier cas (amendes et, également, les ordres de cesser les travaux, les avis d'infractions, l'application du droit de refus, les procédures de conciliation, etc.), nous nous trouvons dans le champ des violations à des normes relatives à la prévention des accidents de travail, normes à caractère disciplinaire qui se trouvent énoncées dans une législation particulière (législation de «santé et sécu-

25. M. Grandbois, op. cit., note $21, i$.

26. Voir Commission de réforme du droit du Canada, les Crimes contre l'environnement, document de travail, CRDC, 1985.

27. K. Lippel, op. cit., note 21, $i$.

28. Il s'agit de la Loi concernant les responsabilités des accidents dont les travailleurs sont victimes dans leur travail et la réparation des dommages qui en résultent, S.Q., 1909, c. 66. En ce qui concerne la théorie du risque professionnel, voir B. Mattei, cité par K. Lippel, op. cit., note $21, i i$. 
rité»); dans le deuxième cas (réparations), on fait référence aux barèmes d'indemnisation qui sont fixés dans un autre type de législation (législation d'«accidents de travail») fondée, à son tour, sur une logique essentiellement restitutive. Certes, des distinctions de cet ordre peuvent et doivent être faites par rapport à n'importe lequel des domaines figurant au tableau 1. Nous estimons, cependant, qu'elle prend ici une connotation toute particulière en raison du fait que ces deux logiques se trouvent à être mises en œuvre, dans le domaine qui nous occupe, par une seule et unique institution, la Commission de la santé et de la sécurité au travail du Québec.

En ce qui concerne les infractions statutaires, il convient de souligner que leur constat ainsi que leur (plus qu') éventuelle mise en forme dans le cadre de procédures judiciaires prennent de plus en plus les apparences d'une mise en scène bureaucratique dont on est en droit de se demander si elle peut avoir une quelconque fonction régulatrice. Certaines données apportées par Lippel ${ }^{29}$ pourront, nous semble-t-il, convier plus d'un lecteur à y réfléchir. Pour ce qui est des indemnisations qu'un travailleur accidenté, ou sa famille en cas de décès, peut réclamer au fonds mutuel d'assurances de la C.S.S.T., rappelons que, contrairement à une croyance assez répandue, elles ne sont pas automatiquement accordées, suite à la première constatation (par un médecin) de l'existence de dommages corporels ou d'une maladie professionnelle. Cette constatation peut en effet n'être que le début d'une interminable bataille médicale où l'entrée en scène des experts de la partie patronale risque souvent de déséquilibrer les rapports de force. Deuxième illusion à défaire: l'affirmation qui soutient que ce fonds d'indemnisation est entièrement financé par l'ensemble d'employeurs n'est en fait qu'une figure de style du genre qu'affectionnent les comptables agréés. En termes économiques, il est plus juste d'affirmer que l'ensemble des consommateurs assume une partie considérable de ce financement en raison du transfert (total ou partiel) des dépenses de cotisation, par l'employeur, à ses coûts de production.

\section{LES CONDITIONS DE POSSIBILITÉ DE L'IMPUNITÉ PÉNALE}

Le but de cette troisième et demière partie de l'article est celui de faire état, de manière forcément schématique, d'un certain nombre de dimensions intégrantes de ce que l'on pourrait appeler le processus de construction sociale, politique et juridique de l'impunité pénale en matière d'illégalismes privilégiés. Certaines de ces dimensions ont déjà

29. K. Lippel, op. cit., note 21, ii. 
fait l'objet d'examens assez poussés dans le cadre de recherches menées au cours des quinze dernières années. D'autres furent à peine effleurées, dans des études d'ailleurs récentes. Cela signifie, en d'autres termes, que la compréhension du rôle qu'on leur attribue est, dans certains cas, encore fort embryonnaire. Quoi qu'il en soit, il apparait de plus en plus évident que toute réflexion qui prétend appréhender, dans toute sa complexité, la question de l'impunité pénale, devra non seulement s'attarder à examiner l'ensemble de ces dimensions mais elle devra, également et surtout, s'employer à comprendre la nature des rapports qu'elles entretiennent entre elles. En d'autres termes, ces dimensions sont les éléments constitutifs d'un objet particulier d'investigation de notre discipline qui, malheureusement, n'a pas toujours reçu l'attention qu'à mon avis son importance réclame.

Il s'agit des dimensions idéologique, matérielle et juridique que nous examinerons, à tour de rôle, dans les pages qui suivent.

\subsection{DIMENSION IDÉOLOGIQUE}

On se retrouve ici dans un terrain qui a déjà fait l'objet d'un bon nombre de recherches en criminologie, soit celui des représentations sociales autour du crime, du criminel et de la répression pénale ${ }^{30}$. Il est cependant permis d'affirmer que l'objet particulier qui nous intéresse n'occupe qu'une place relativement modeste à l'intérieur de cet effort d'investigation ${ }^{31}$. Pourtant, il est relativement facile d'admettre, du moins en principe, que les représentations sociales (véhiculées dans les divers moyens de communication, dans les échanges de la vie quotidienne, etc.) jouent un rôle qui est sans doute loin d'être négligeable dans la genèse des règlements de conflits qui ont cours en matière d'illégalismes privilégiés. Autrement dit, les rapports que nous établissons avec les événements relevant des différents domaines que nous venons d'examiner tendent, le plus souvent, à les confiner dans une catégorie de représentation de la réalité conflictuelle autre que celle où logent les affaires criminelles, et cela en dépit du fait qu'il s'agisse ou non d'un type d'événement passible d'une prise en charge pénale ou

30. Voir, entre autres, C. Faugeron et al., De la déviance et du contrôle social, rapport de recherche, Paris, D.G.R.S.T., 1975; C. Faugeron, «Du simple au complexe : les représentations sociales de la justice pénale», Déviance et société, 2, (4), 1978, pp. 411432; Ph. Robert, C. Faugeron, la Justice et son public. Les représentations sociales du système pénal, Genève, Masson/Médecine et Hygiène, 1978.

31. Au sujet des représentations de la délinquance d'affaires, dans la presse écrite, voir, par exemple, M.F. Armand, P. Lascoumes, «Malaise et occultation : perceptions et pratiques du contrôle social de la criminalité d'affaires», Déviance et société, $I$, (2), 1977, pp. 135-169. 
même d'un événement qui a déjà fait l'objet de cette forme d'intervention. Sans vouloir entrer dans les nombreuses considérations que cette question suscite, nous nous permettrons cependant d'indiquer deux de ses principales voies d'approche.

La première, que l'on trouve dans certaines recherches sur la délinquance d'affaires, met particulièrement l'accent sur le fait que c'est à des personnes morales et non à des personnes physiques que les simples individus se trouvent fréquemment confrontés dans la trame de ces événements. Or, étant donné que la représentation classique (même au sens thêtral du terme, si l'on veut) de l'acte criminel oppose, avec une farouche tenacité, deux individus, deux rôles qu'on ne saurait confondre (agresseur $v s$ victime), on comprendrait la difficulté de la transposition d'une telle mise en scène dans le cadre d'un événement qui met face à face l'individu et une corporation. Et dire que l'individu fait face à une corporation c'est déjà aller vite en besogne, empressonsnous de l'ajouter. La plupart du temps, en effet, la mise en opposition de l'individu à la réalité corporative est hautement problématique, vu le nombre considérable de réalités infiniment plus simples, de la vie quotidienne, qui médiatisent ce rapport. (C'est le médicament nocif qui est dans le champ de son expérience, et non les innombrables étapes de sa production et mise en marché; c'est le marchand d'essence indépendant que le consommateur rend responsable de la montée des prix, et non les pratiques de concurrence déloyale des grandes compagnies pétrolières.)

Deuxième voie d'approche. La difficulté d'appréhension des événements impliquant des pratiques corporatives, à partir d'un code aprioriste de représentation des transgressions à la loi pénale, peut également être visualisée au plan du langage courant et, souvent même, technique. L'un des termes sans doute le plus utilisé pour désigner des événements, aux conséquences parfois extrêmement graves, qui relèvent de certains des domaines que nous avons examinés est celui d'«accident». Des accidents de travail jusqu'aux fuites accidentelles de produits toxiques ou aux accidents nucléaires, en passant par les accidents de parcours qui stoppent parfois brusquement l'ascension un peu trop rapide de certains hommes d'affaires, on voit défiler une séquence pour le moins hétérogène d'événements dont le seul point en commun est celui d'être dus aux insondables tracasseries du destin. C'est qu'à force de banaliser la signification de ce terme, comme nous le faisons dans le langage quotidien, comme nous l'ont peut-être appris à faire les médias d'information, on finit par oublier qu'en s'en servant, on se prononce déjà en quelque sorte sur le caractère imprévisible de l'événement en question. Dans le même ordre d'idées, et ceci est particulière- 
ment visible dans la presse écrite, on ne parlera pas non plus de faute, mais de maladresse, de bavure, de malchance. On se permettra, à l'occasion, de dire «fraude», ce qui n'est pas à proprement parler un exploit linguistique vu qu'il est courant de dire "fraude scolaire», «fraude électorale», etc., quoique les formules «abus de pouvoir économique» et «profits excédentaires» semblent récolter une plus grande faveur des bâtisseurs de l'opinion publique. Et à l'allégation qui prétend que les organes d'information doivent bien se garder de se prononcer sur la nature juridique des événements qu'ils rapportent, il suffit de rappeler qu'il est plutôt rare qu'ils le fassent, par exemple, lorsqu'il est question d'atteinte à la vie dont les formes sont déjà cristallisées dans des représentations collectives. La pratique macabre des quotidiens de tenir une comptabilité sur les «meurtres» de l'année, alors que l'éventuel «meurtrier» est, dans la pire des hypothèses, introuvable, et dans la meilleure, à sa première comparution devant un juge au moment même où le périodique circule, illustre bien le peu de fondement d'une telle allégation.

\subsection{DIMENSION MATÉRIELLE}

L'impunité pénale des illégalismes privilégiés doit également être examinée sous l'angle de certaines de leurs caractéristiques matérielles. En d'autre termes, le fait qu'ils se montrent à ce point réfractaires à la prise en charge pénale est dû, en partie, à l'agencement d'un certain nombre de facteurs situés au plan phénoménologique. Cela peut être constaté à au moins trois différents niveaux.

i) Occultation matérielle - La complexité technique des opérations qui ont cours dans les différents domaines que nous avons examinés, ainsi que les réseaux de complicité où d'innombrables intervenants s'enchaînent les uns après les autres, constituent probablement les facteurs le plus souvent invoqués pour rendre compte des difficultés de repérage des illégalismes privilégiés. Mais s'il est un facteur qui assure, indépendamment de tout autre, la visibilité précaire dont jouissent ces illégalismes c'est bien le fait que les événements d'où ils découlent se constituent, invariablement, dans des espaces sociaux relativement bien protégés, ou carrément soustraits, des regards indiscrets. Les bureaux (d'affaires, des agences gouvernementales), les installations des usines, les laboratoires, les grands chantiers de travail, voilà autant de lieux de la vie sociale dont l'occupation et l'usage se trouvent soumis à des règles bien établies, règles d'ailleurs reconnues soit par le silence, soit par la lettre des lois. Soit dit en passant, la référence au degré de protection de l'espace social nous semble plus appropriée que le recours à la 
distinction, plus rigide, entre espace public (champ des illégalismes populaires) et espace privé (champ des illégalismes bourgeois) dont se servent certains auteurs ${ }^{32}$, pour expliquer la difficile visibilité de ces illégalismes.

ii) Lien de causalité - La difficulté voire l'impossibilité totale d'établir, en termes scientifiques, un lien de cause à effet entre un dommage constaté et un nombre important de pratiques qui relèvent des domaines que nous considérons est à la fois un aspect particulier de la question soulevée plus haut et un facteur en soi d'exclusion de ces pratiques du champ de la responsabilité pénale et même civile. Certains travaux récents de la Commission de réforme du droit du Canada non seulement illustrent bien l'importance de cette question comme ils proposent d'excellents éléments de réflexion sur la nature des rapports que certains secteurs du monde scientifique entretiennent avec les grandes corporations ${ }^{33}$.

iii) Réseau de contrôle - Le fait que les illégalismes privilégiés disposent en permanence d'un réseau de contrôle aux modalités multiples d'intervention est, bien entendu, un facteur qui assure la reproduction constante des conditions de possibilité de leur exclusion du champ pénal. La question de savoir si l'exclusion que l'on constate s'explique, en partie, par l'existence d'un tel réseau ou si c'est plutôt l'inverse qui se vérifie ne saurait être convenablement examinée que dans le cadre d'une étude à part, à vocation essentiellement génétique.

\subsection{DIMENSION JURIDIQUE}

Créer des lois c'est créer, en même temps, la possibilité d'y désobéir, disait Sartre dans Saint Genet, comédien et martyre... et d'y échapper, on pourrait sans doute ajouter. Ce qui revient à dire que l'impunité pénale loge avant tout dans la loi pénale elle-même, ou alors on serait en droit de se demander en quoi consiste la spécificité de cette forme d'exclusion. Constatation banale, certes, à la condition que l'on s'obstine à rappeler, jusqu'à l'ennui, par une telle proposition, que la loi pénale possède d'importantes «lacunes» au plan substantif. Or, cette conception, en termes de pure négativité, de l'impunité pénale, con-

32. Voir, par exemple, Ph. Robert, «Au thêâtre pénal. Quelques hypothèses pour une lecture sociologique du «crime», Déviance et société, 9. (2), 1985, pp. 89-105.

33. Voir T.F. Schrecker, op. cit, note 22; Commission de reforme du droit du Canada, la Pollution en milieu de trovail, document de travail, CRDC, Ottawa, 1987; Commission de réforme du droit du Canada, les Pesticides au Canada; étude de la législation et de la politique fédérales, document d'etude, CRDC, Ottawa, 1987; J. Braithwaite, op. cit., note 19. 
ception d'ailleurs courante, nous empêche peut-être de voir que ce n'est pas dans ce qu'elle tait mais plutôt dans ce qu'elle énonce que la loi pénale restreint véritablement son champ effectif d'intervention. En effet, si l'on accepte que ce terme doit désigner, à la fois, un ensemble de prescriptions relatives à un certain nombre de conduites et un ensemble de règles concernant la mise en ceuvre de ces prescriptions, on peut identifier des facteurs d'exclusion non seulement au plan substantif de la loi pénale mais, également, $i$ ) au plan des règles de procédure (les ajournements successifs des séances, les rejets des dénonciations, les arrêts des procédures, les abandons des poursuites, etc.); ii) au plan des règles relatives à l'établissement de la preuve (les obstacles à l'admissibilité des preuves relatives à des actes dits astucieux ou à haut degré de complexité technique) et, iii) au niveau des principes qui guident la mise en forme des infractions criminelles par les divers opérateurs situés à différentes étapes du processus judiciaire (c'est-à-dire des principes qui orientent, à chaque étape de la procédure pénale, des choix qui vont s'avérer décisifs pour la suite du processus; à travers ces choix, ces opérateurs peuvent donner, à une même affaire, différentes configurations à la lumière de la loi pénale, y compris, bien entendu, celles qui excluent toute possibilité d'allégation de responsabilité criminelle ${ }^{34}$.

\section{CONCLUSION}

S'il est un but, avant tout autre, qui a guidé les propos que j'ai tenus dans cet article, c'est bien celui de faire la preuve de l'importance et de la nécessité pour la criminologie québécoise d'investir (ou de réinvestir, selon le cas) les domaines que sommairement $j$ 'indique. Et je prétends que la relative urgence de le faire se justifie, entre autres, par le fait que l'élan qui a animé les premières tentatives d'investigation en cette matière, depuis la fin de la dernière décennie, semble s'être estompé au cours des dernières années. D'autre part, je me suis également employé à démontrer que les questions qui ont été ici soulevées s'avèrent suffisamment importantes, au plan théorique, pour pouvoir justifier que l'on y réfléchisse dans un tout autre but que celui de simplement dénoncer la réalité qu'elles circonscrivent. En effet, le ton dénonciateur qui a marqué, et qui marque encore aujourd'hui, un certain nombre de travaux engagés dans cette voie a contribué à escamoter le fait que, derrière la réalité de l'exclusion d'un conflit du champ de l'intervention pénale, se tisse une trame complexe de rapports (de collaboration, de confrontation) entre différents systèmes normatifs. Ou, en

34. À ce sujet, voir F. Acosta, op. cit., note 14, iv. 
d'autres termes, que cet objet ne soulève vraiment d'importantes questions théoriques que dans la mesure où on le construit et on le problématise en tenant compte de sa positivité. On s'est peut-être trop souvent contenté de concevoir le pénal, son discours, les pratiques institutionnelles qu'il constitue, comme un objet étanche. Sans qu'il soit nécessaire, au contraire, de mettre en question sa spécificité, il apparaît de plus en plus évident que l'on gagne à inscrire cet objet particulier dans le cadre d'une problématique plus large qui tienne compte de la multiplicité des formes de règlements de conflits et, surtout, des rapports complexes qui se nouent entre elles.

Néanmoins, je suis bien forcé d'admettre que ce n'est pas à coup de bonnes intentions théoriques que l'on arrive à faire accepter l'idée qu'il est possible de se pencher sur de telles questions sans pour autant en faire la tribune d'un plaidoyer en faveur de l'élargissement des assises de la répression pénale. Et à plus forte raison lorsque le fantôme de la criminalisation hante certaines pratiques relatives aux domaines que nous avons examinés. La proposition récente de la Commission de réforme du droit du Canada d'inclure, dans le Code criminel, une nouvelle infraction sous le titre de «crime contre l'environnement» en est d'ailleurs un exemple de taille ${ }^{35}$. Par ailleurs, il n'est pas rare de nos jours d'entendre des propositions semblables, qui ne bénéficient toujours pas de la même autorité que celles avancées par la Commission, à l'égard de différentes formes d'illégalismes des domaines ici mentionnés. Autant dire que l'occasion semble belle pour qu'on s'y prononce dans le cadre de notre discipline. Dans le but de contribuer à un débat dont la nécessité ne fait aucun doute dans mon esprit, j'énumère ici quatre arguments qui me semblent démontrer le caractère profondément illusoire de toute démarche de criminalisation en cette matière.

i) Le pénal comme réserve de pouvoir - Il faudrait faire preuve d'une certaine naiveté pour penser que le fait de criminaliser, au plan formel, une ou plusieurs conduites qui relèvent des domaines que nous avons ici examinés puisse éliminer le recours à d'autres modes de règlement des conflits. Au contraire, il n'est pas déraisonnable de croire que, dans certains cas précis, le spectre de la sanction pénale puisse être utilisé comme un moyen d'imposer, et par conséquent de consolider dans les usages, une autre voie de règlement. Des manifestations de cette tendance ont pu déjà être observées dans le domaine de ce que les

35. Voir Commission de réforme du droit du Canada, op. cit., note 26. 
milieux policiers appellent le «contrôle de la criminalité économique ${ }^{36}$ ».

ii) Les foyers de résistance - Dans d'autres domaines (nous pensons ici, particulièrement, à ceux de la santé publique, de l'environnement et, peut-être, de la santé et de la sécurité au travail), une politique de criminalisation risque plutôt de se heurter à des résistances farouches des opérateurs des agences de contrôle et produire, ainsi, l'effet en quelque sorte contraire de celui que nous venons de décrire. En effet, certaines recherches ont déjà amplement démontré qu'un bon nombre de ces opérateurs entretiennent des rapports de collaboration avec les secteurs qu'ils doivent contrôler; d'ailleurs, dans certains cas on peut aller jusqu'à dire qu'ils en dépendent carrément pour l'exercice de leurs fonctions ${ }^{37}$. Une telle situation s'explique, cela va de soi, sauf peut-être pour ceux qui se complaisent dans une vision idyllique des fondements des rapports sociaux, par l'extraordinaire situation de force dans laquelle se trouvent les corporations qui opèrent dans les secteurs que ces opérateurs doivent contrôler. Dans un tel contexte, il est possible d'admettre que l'entrée en scène de la loi pénale puisse être reçue comme un obstacle à leur intervention suffisamment fort pour pouvoir :

- dans certains cas, donner lieu à des mesures visant à ralentir considérablement leurs activités;

- dans d'autres, confirmer le bien-fondé du caractère gestionnaire de leur intervention et ainsi justifier le fait qu'ils ne réfèrent à la justice pénale qu'un nombre vraisemblablement très restreint, et choisis, de cas.

iii) Les transferts difficiles - La deuxième hypothèse ci-haut, réclame d'ailleurs une précision importante. En effet, la possibilité qu'une éventuelle politique de criminalisation en matière d'illégalismes privilégiés s'accompagne de mesures effectives, matérielles en particulier, qui assurent sa mise en application est pour le moins douteuse. Ce qui revient à dire que l'appareillage administratif qui assume

36. Certains dossiers examinés à l'escouade des crimes économiques de la Sûreté du Québec, dans le cadre d'une recherche sur le contrôle de la criminalité d'affaires au Québec (voir Rico et al., op. cit., note 14, ii), portaient des traces évidentes de cette pratique. Les dossiers en question étaient le résultat d'enquêtes portant sur des allégations d'actes de corruption par des fonctionnaires d'organismes gouvernementaux auxquels leurs supérieurs hiérarchiques avaient offert des garanties d'impunité en échange soit de leurs démissions, soit du remboursement des sommes indóment perçues, soit des deux. Il est évident, dans ces cas, que les ententes proposées ne se sont pas concrétisées, pour des raisons d'ailleurs que les dossiers en question ne laissaient pas clairement entrevoir.

37. C'est ce que démontrent, entre autres, Schrecker, op. cit, note 22 et Braithwaite, op. cit., note 19. 
aujourd'hui les tâches de constatation, d'enregistrement et de mise en forme de ces illégalismes devra de toute évidence rester en place. Une pareille situation de dépendance de l'appareil judiciaire à l'égard de sa contrepartie administrative pourra se traduire en pratique par la situation suivante: les instances judiciaires pénales ne seront saisies que des affaires qui leur seront occasionnellement transmises par les agences administratives de contrôle. Or, rien ne nous assure, comme l'a très bien fait remarquer Lascoumes ${ }^{38}$, qu'une affaire construite selon les paramètres d'une logique administrative d'intervention réponde nécessairement aux critères de recevabilité juridique qui commandent la mise en marche de la prise en charge pénale. Et à plus forte raison lorsque l'affaire en question possède des dimensions techniques relativement importantes.

iv) L'équité perverse - Dernière raison, et pas la moindre, bien au contraire, il serait sûrement mal avisé de voir dans la création de ces nouveaux crimes un moyen d'atteindre un «certain équilibre punitif», et il m'apparaît inutile de $m$ 'engager ici dans une longue démonstration au sujet du caractère irrémédiablement pervers d'une telle conception de l'équité. À ce titre, je me contenterai tout simplement d'affirmer que, jusqu'à preuve du contraire, je ne vois que deux effets à escompter de toute initiative de criminalisation en cette matière : effet de légitimation des pratiques répressives actuellement en vigueur, d'une part, effet d'élargissement du champ déjà occupé par ces pratiques, de l'autre.

En ce qui concerne ce deuxième effet, je tiens à souligner que malgré tous les obstacles que (de bon cceur) je constate à la mise en application d'une politique de criminalisation des illégalismes privilégiés, des exceptions à ce scénario demeurent possibles. Et c'est probablement là que les véritables enjeux de ce dangereux pari de politique criminelle se jouent. Car il m'apparaît qu'il y a de gros risques à oublier que le monde des affaires est aussi le monde des petites entreprises familiales, acculées souvent à l'insolvabilité, si ce n'est à la tentation de la banqueroute, par les pratiques illégales des géants corporatifs. Que l'administration publique est aussi, et surtout, le monde de milliers de modestes fonctionnaires. Tout comme d'ailleurs la santé publique est aussi une affaire de professionnels de très bas échelon, l'environnement, un gagne-pain pour bien des camionneurs plus ou moins au fait de la toxicité des produits qu'ils transportent, les chantiers de travail, un terrain occupé par un grand nombre de petits entrepreneurs de la

38. Voir $i$ ) P. Lascoumes, op. cit., note 3, $i$; ii) P. Lascoumes, Vemeuil, Délit fiscal et tou délit pénal: les poursuite en matière fiscale, une étude d'interface, Paris, Service d'études pénales et criminologiques, 1981. 
construction. Autant de protagonistes en puissance, pourrait-on dire, pour la grande répétition générale à laquelle nous a habitué, il y a belle lurette, l'appareil pénal. 